\title{
Stellar magnetic fields: The view from the ground and from space
}

\author{
Gregg A. Wade \\ Department of Physics, Royal Military College of Canada, P.O. Box 17000 Station Forces, \\ Kingston, ON, Canada K7K 7B4 \\ email: wade-g@rmc.ca
}

\begin{abstract}
This article reviews the methods of measurement which allow us to infer the presence of magnetic fields in (A) stars. Beginning with the basic observational consequences of the Zeeman effect, we describe various modern spectroscopic and polarimetric techniques which allow us to directly detect and characterise magnetic fields in stellar photospheres. Sometimes, nature conspires to make such detections difficult, forcing us to rely on indirect (proxy) indicators of magnetism. This talk will also briefly discuss a number of these indirect field indicators, some of which demand space-based observations.
\end{abstract}

Keywords. Methods: data analysis, techniques: polarimetric, techniques: spectroscopic, stars: magnetic fields

\section{Introduction}

We are gathered here in Poprad to discuss "The A-Star Puzzle", the scientific issues associated with stars of spectral type A, with effective temperatures between about 7500 and $10000 \mathrm{~K}$. If we draw vertical lines on the HR diagram according to these temperature extremes, we see that "A stars" include a tremendous variety of objects, on various evolutionary paths. At the earliest evolutionary stages, we have pre-Main Sequence A stars, both those which remain enshrouded in their nascent cocoons (pre-birthline) and those which have recently shed their formative gas and dust clouds (post-birthline) and are observable in visible light. These stars are significantly reddened, are typically rapidlyrotating, and exhibit emission lines due to remaining circumstellar matter. On the Main Sequence, the A stars include chemically peculiar stars, both magnetic and nonmagnetic, as well as pulsating variables and "normal" stars. These stars exhibit a tremendous variety of physical and spectral characteristics: line profile shape and polarisation variations, peculiar energy distributions, vertically and laterally non-uniform abundance distributions, and strong magnetic fields. Evolved A stars include higher-mass supergiants, whose spectra are dominated by the effects of mass-loss and wind variability, and intermediatemass giants which include the radially, and possibly non-radially, pulsating RR Lyrae stars.

The huge variety of physical properties of A-type stars makes the potential presence of magnetic fields in their atmospheres and immediate circumstellar environments extremely interesting. At the same time (and as we shall see in the following sections), the large range of spectroscopic characteristics demands a sensitive and flexible suite of techniques for detecting and characterising such fields.

\section{Overview of the Zeeman effect in spectral lines}

Stellar magnetic fields are detected and characterised using the Zeeman effect. When an atom is immersed in an external magnetic field $B$, individual atomic levels (with energy 

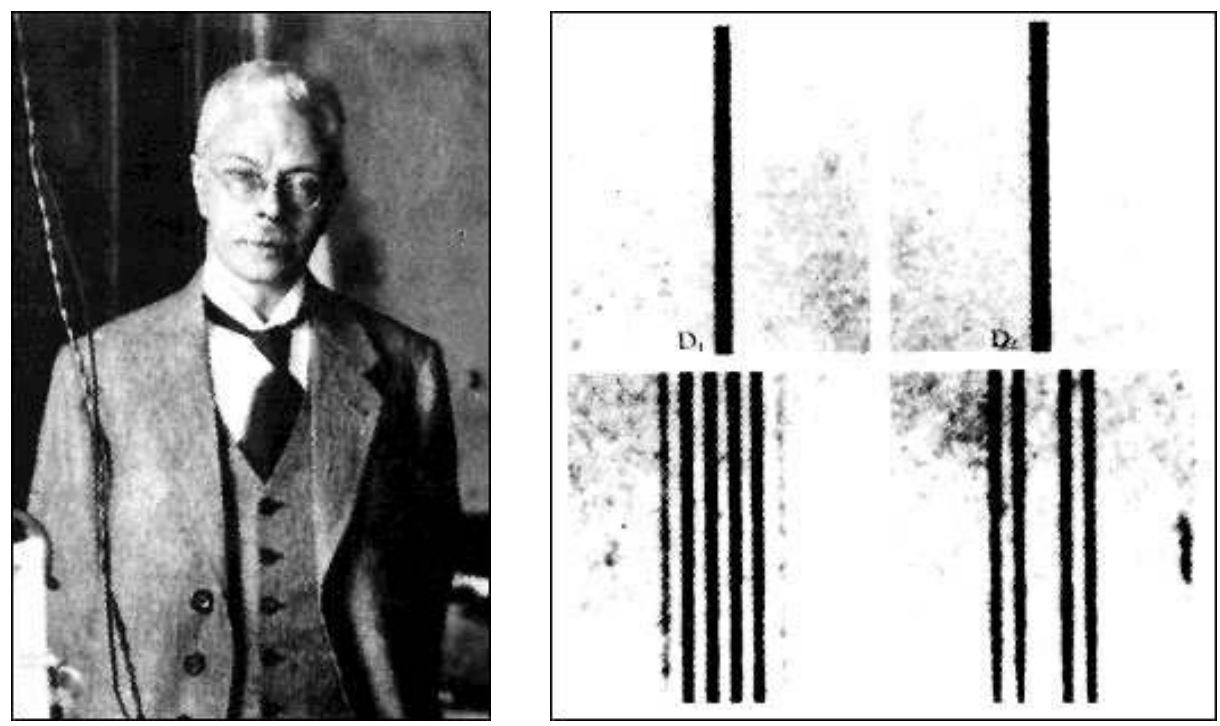

Figure 1. Left: Dr. Pieter Zeeman, discoverer of the effect to which his name is attached. Right: Photograph of the splitting of the Na D doublet under the influence of an external magnetic field, as reported by Zeeman (1897).

$E_{0}$, total angular momentum $J$, and Landé factor $\left.g\right)$ are split into $(2 J+1)$ substates, characterised by their magnetic quantum number $M(-J \leqslant M \leqslant+J)$. The energies of these atomic states are given by:

$$
E(M)=E_{0} g M \hbar \omega_{L}
$$

where $\omega_{L}=e B /\left(2 m_{e} c\right)$ is the Larmor frequency (e.g., Mathys 1989).

As a result of this splitting, each transition that generates a single line in the stellar spectrum when no field is present leads, in the presence of a field, to a group of closelyspaced spectral lines, or Zeeman components. For transitions in LS coupling, these lines may be grouped into two different types with different properties. Those lines resulting from transitions in which $M$ does not change (i.e. $\Delta M=0$ ) are spread symmetrically about the zero-field wavelength $\lambda_{0}=h c / E_{0}$ of the line. These are called the $\pi$ components. Those resulting from transitions in which $\Delta M= \pm 1$ have wavelengths to the red $(+)$ and blue $(-)$ of the zero-field wavelength. These are called the $\sigma$ components. The wavelength separation between the centroids of the $\pi$ and $\sigma$ component groups can be calculated from Eq. (2.1), and is:

$$
\Delta \lambda=\frac{e N \bar{g}}{4 \pi m_{e} c^{2}} \lambda_{0}^{2} \equiv \Delta \lambda_{Z} B \bar{g}
$$

where $\bar{g}$ is the effective Landé factor of the transition and $\Delta \lambda_{Z}$ is called the Lorentz unit. Note that the splitting is directly proportional to the strength of the applied field $B$. For illustration, a $5 \mathrm{kG}$ magnetic field produces a splitting of $\sim 0.1 \AA$ at $5000 \AA$.

Pieter Zeeman was the first to report these splitting properties in his seminal paper of 1897, The Effect of Magnetisation on the Nature of Light Emitted by a Substance:

Sodium was strongly heated in a tube of biscuit porcelain, such as Pringsheim used in his interesting investigations upon the radiation of gases. The tube was closed at both ends by plane parallel glass plates.... The tube was placed horizontally between the poles, at right angles to the lines of force. The light of an arc lamp was sent through. The absorption spectrum showed both D lines. The tube was 


\section{LOCAL CASE}
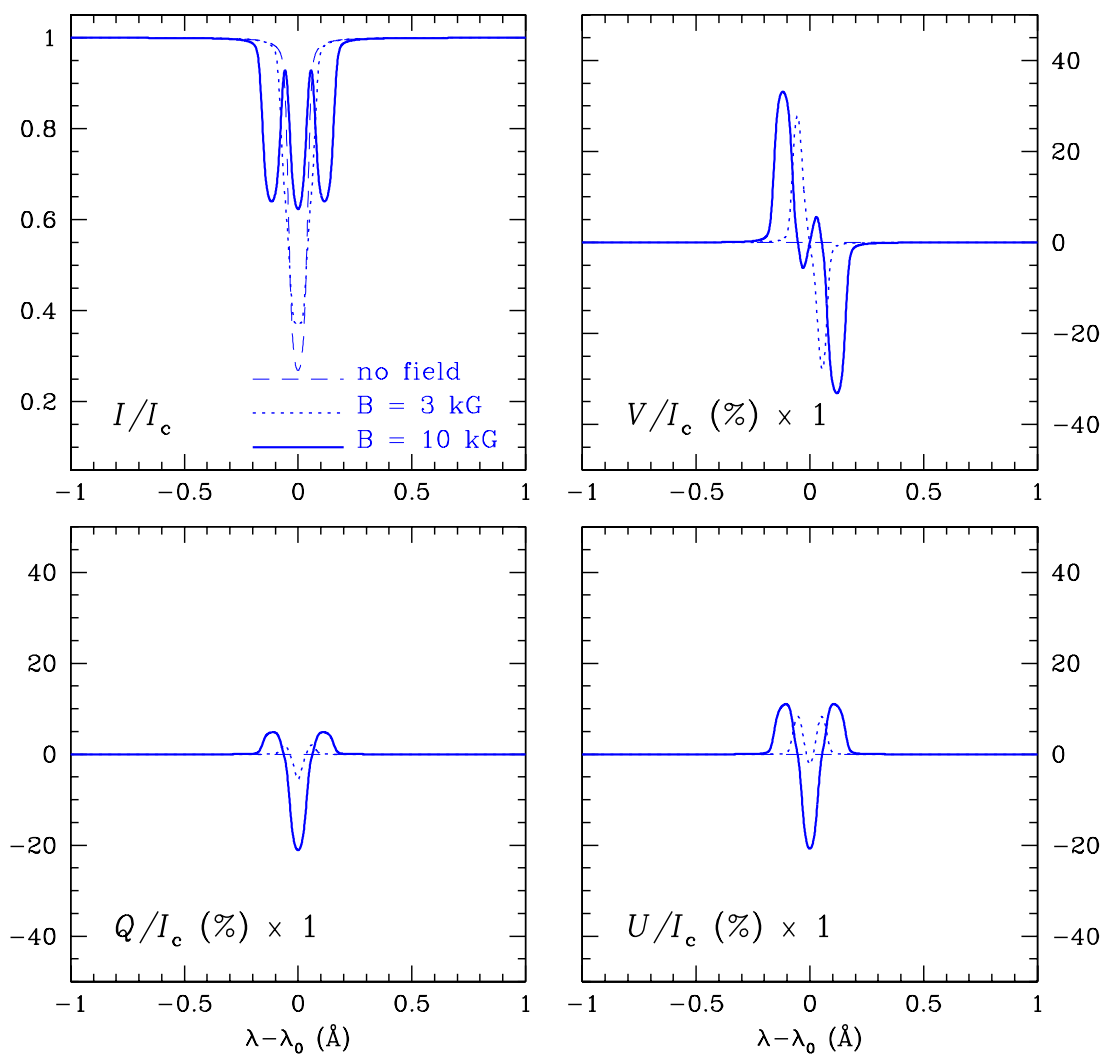

Figure 2. Influence of the intensity of the applied magnetic field on a spectral line, in natural light (Stokes $I$ ), circular polarisation (Stokes $V$ ) and linear polarisation (Stokes $Q$ and $U$ ). Each set of these "local" profiles corresponds to a single intensity and direction of the magnetic field, as might be observed in a laboratory or on the sun. Profiles are shown for 3 different field intensities.

continuously rotated round its axis to avoid temperature variations. Excitation of the magnet caused immediate widening of the lines. It thus appears very probable that the period of sodium light is altered in the magnetic field.

(Zeeman 1897)

Zeeman and his observations are illustrated in Fig. 1. At the suggestion of Hendrik Lorentz, Zeeman furthermore investigated the polarisation properties of the $\pi$ and $\sigma$ components. Zeeman found that in a magnetic field aligned parallel to the observer's line-of-sight (a longitudinal field), the $\pi$ components vanish, whereas the $\sigma$ components have opposite circular polarisations. On the contrary, when the magnetic field is aligned perpendicular to the observer's line-of-sight (a transverse field), the $\pi$ components are linearly polarised parallel to the field direction, while the $\sigma$ components are linearly polarised perpendicular to the field direction:

I have since found by means of a quarter-wave plate and an analyser, that the edges of the magnetically-widened lines are really circularly polarised when the line of sight coincides in direction with the lines of force... On the contrary, if one looks at the flame in a direction at right angles to the lines of force, then the edges of the broadened sodium lines appear plane polarised, in accordance with theory.

(Zeeman 1897)

In summary, Zeeman observed that application of a magnetic field leads to the splitting 


\section{STELLAR CASE (DIPOLAR FIELD)}
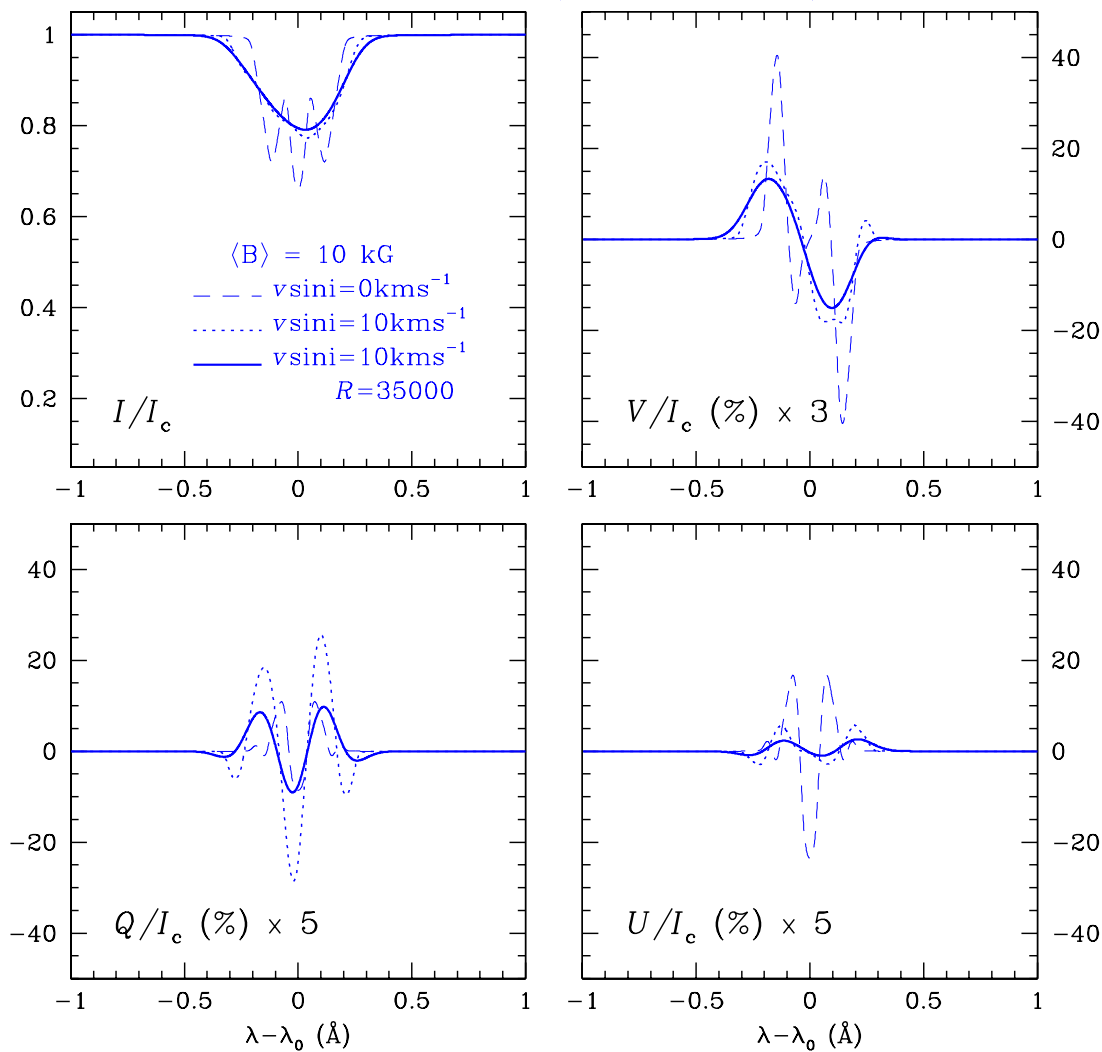

Figure 3. Disc-integrated line profiles (solid and dotted lines) as might be encountered in the spectrum of an unresolved star with a distribution of field strengths over the observed hemisphere (in this case, a dipole corresponding to a mean integrated field modulus of $10 \mathrm{kG}$ ). Profiles are shown for $v \sin i=0 \mathrm{~km} \mathrm{~s}^{-1}, v \sin i=10 \mathrm{~km} \mathrm{~s}^{-1}$, and $v \sin i=10 \mathrm{~km} \mathrm{~s}^{-1}$ with additional instrumental broadening corresponding to a spectral resolution of $R=35000$. Note the significant decrease in the diagnostic content of the Stokes $I$ profile, and the decrease in the amplitude of the polarisation profiles, as a consequence of disc integration and rotation.

of spectral lines into multiple Zeeman components, and that the components are polarised in particular ways corresponding to the orientation of the field. Thus, both the intensity and the geometry of the magnetic field in the line-forming region are encoded in the line profile as a consequence of Zeeman effect.

In the "weak-field limit", when the ratio of the Zeeman splitting to the intrinsic width of the line $\Delta \lambda_{Z} / \Delta \lambda_{I} \ll 1$, under the assumption of a linear Milne-Eddington source function, one obtains the first-order solution for the Stokes profiles emergent from a stellar photosphere:

$$
\begin{aligned}
I(\tau=0, \lambda) & =B_{0}+B_{0} \beta_{0} \mu[1+\eta(\lambda)]^{-1} \\
Q(\tau=0, \lambda) & =0 \\
U(\tau=0, \lambda) & =0 \\
V(\tau=0, \lambda) & =-\bar{g} \Delta \lambda_{Z} B_{Z} \frac{d I(\lambda)}{d \lambda}
\end{aligned}
$$

where $B_{0}$ and $\beta_{0}$ are the Milne-Eddington parameters, $\mu$ is the limb angle, $\eta(\lambda)$ is the 
line opacity variation in the absence of a magnetic field, and $B_{Z}$ is the longitudinal component of the magnetic field.

Eqn. (2.3) will be immediately recognised as the Milne-Eddington solution for the emergent Stokes $I$ profile in the absence of a magnetic field, i.e., to first order, the Stokes $I$ profile is unaffected by the presence of a weak magnetic field. Furthermore, Eqs. (2.4) and (2.5) show, to first order, that the line profile is not linearly polarised for weak fields. In fact, the only first-order influence of the magnetic field in the weak-field regime is on the amplitude of the Stokes $V$ profile. For any spectral line, the shape of Stokes $V$ is determined entirely by the shape of the corresponding Stokes $I$ profile, via the derivative $d I / d \lambda$. However, the amplitude of Stokes $V$ is proportional to the intensity of the longitudinal component of the magnetic field. This implies that for most stars, the most easily accessible Zeeman diagnostic will be line circular polarisation, and for such stars only the longitudinal field component can be practically measured. The linear polarisation Stokes parameters, which constrain the transverse components of the field, will be in general much weaker (appearing only at second order), and consequently much more difficult to detect. Synthetic line profiles, illustrating the influence of field strength on the splitting and polarisation profile amplitudes, are shown in Fig. 2.

In real stars, our ability to diagnose magnetic fields depends on various (nonmagnetic) physical and spectroscopic attributes (e.g., visual magnitude, $S / N$, spectral line depth and density, rotational velocity, spectral resolving power of the spectrograph), as well as the magnetic field. In many situations, the observable signal may be near the practical limits of available instrumentation. This contrast is illustrated in Fig. 3, in which we examine disc-integrated line profiles as they might actually appear in the spectrum of an unresolved, rotating, magnetic star.

\section{Application}

And so, as we have seen in section 2, the Zeeman effect leads to various observational effects in both natural and polarised light. Depending on the particular spectroscopic characteristics of a star, as well as the intensity and the geometry of the magnetic field, some of these effects may provide a sensitive diagnosis of the magnetic field, and some may not.

\subsection{Line broadening, desaturation and splitting in natural light}

In the absence of a polarimetric diagnosis (i.e., in the natural light or Stokes $I$ spectrum), the presence of a magnetic field in the stellar photosphere can be detectable in certain special situations. For magnetic fields sufficiently intense to induce an appreciable separation of the Zeeman components of spectral lines (splitting $\Delta \lambda_{Z}$ of the same order of magnitude as the observed line width $\Delta \lambda_{\text {obs }}$ accounting for all broadening effects, including rotation), broadening or even resolved splitting of spectral lines is possible. Roughly, the field intensity required to produce a resolved splitting is of order $1 \mathrm{kG}$ per $\mathrm{km} \mathrm{s}^{-1}$ of line broadening. Hence, fields in excess of about $3 \mathrm{kG}$ are required to produce a resolved magnetic splitting in Fe lines of a non-rotating A star; a field in excess of $13 \mathrm{kG}$ is necessary to split the lines of an A star rotating at $10 \mathrm{~km} \mathrm{~s}^{-1}$. Therefore broadening and splitting tend only to be observable in the unpolarised spectra of the sharpest-lined A stars, and are essentially only unambiguously detectable for relatively large fields. These effects have been recently exploited in studies by e.g., Leone et al. (2003), Stütz et al. (2003) and Landstreet \& Mathys (2000). Notably, Mathys and collaborators employed these effects in large scale studies of the magnetic properties of Ap stars during the 1990s (Mathys 1995, Mathys et al. 1997). 

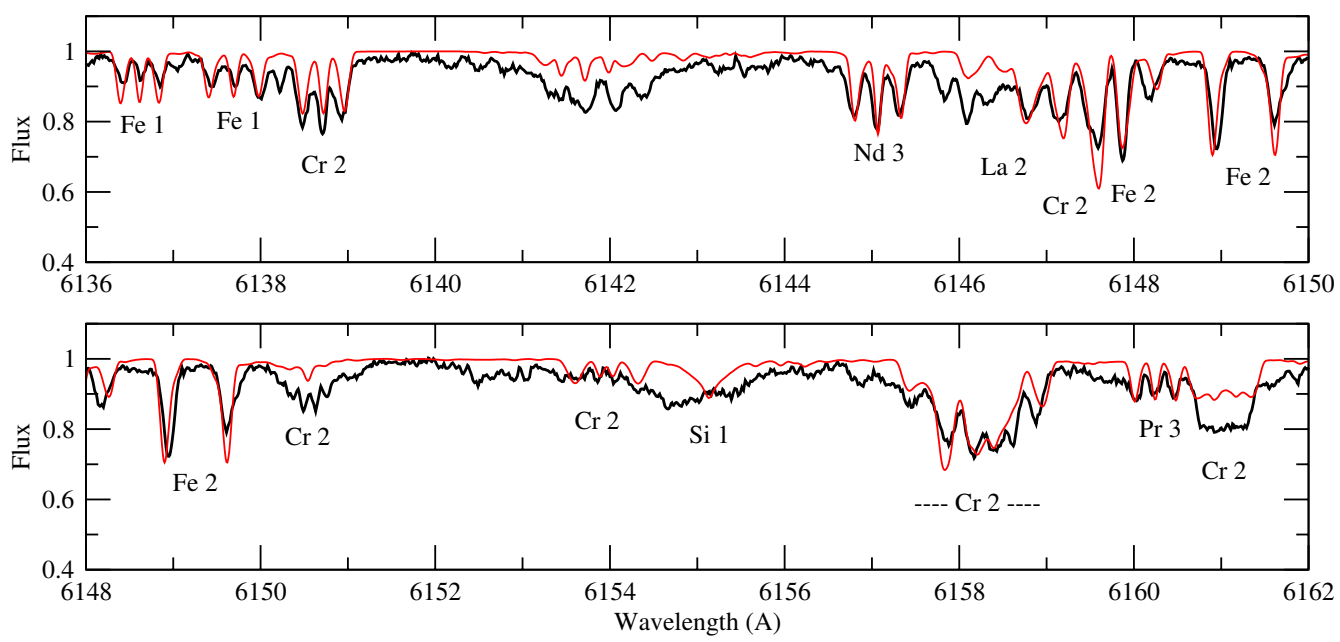

Figure 4. Observed and calculated spectra of HD 66318, reported by Bagnulo et al. (2003). Note the resolved splitting of many lines into Zeeman doublet and triplet patterns.

Fig. 4 illustrates the influence of magnetic splitting on the spectrum of a sharp-lined star. It shows observed (ESO Coudé Echelle Spectrograph) and calculated (ZEEMAN, Landstreet 1988, Wade et al. 2001) spectra of the young cluster Ap star HD 66318 reported by Bagnulo et al. (2003). Note the splitting of most lines into double and triplet Zeeman patterns.

\subsection{Circular polarisation in metal and hydrogen lines}

As discussed in Sect. 2, the most easily accessible polarised diagnostic of stellar magnetic fields is circular polarisation (resulting from the longitudinal Zeeman effect and indicative of the presence of a line-of-sight component of the magnetic field in the stellar photosphere (a longitudinal magnetic field)). Typically, Zeeman circular polarimetry (and linear polarimetry, to be discussed in the section following) is obtained differentially, by recording the two orthogonal polarisation states (left and right circularly polarised light, separated using a $\lambda / 4$ retarder and a polarising beamsplitter) simultaneously on a CCD $†$. The sum of the recorded fluxes gives the natural light spectrum (Stokes $I$ ), whereas their difference gives the net circularly polarised flux (Stokes $V$ ). This procedure is described in some detail by, e.g., Bagnulo et al. (2002).

Zeeman circular polarimetry has the advantage of being sensitive even under conditions of moderate or rapid stellar rotation. Using metal lines, practical sensitivity up to about $60 \mathrm{~km} \mathrm{~s}^{-1}$ can be achieved for Ap stars with strong fields (e.g., Wade et al. 2000a) $\ddagger$. When metallic-line spectropolarimetry is coupled with the powerful Least-Squares Deconvolution multi-line technique (LSD, Donati et al. 1997), remarkably high precision can be attained, particularly for the cooler A stars. Using intrinsically broad lines such as Balmer lines, sensitivity can furthermore be extended to several hundreds of $\mathrm{km} \mathrm{s}^{-1}$ (e.g., Bagnulo et al. 2002, see also, e.g., Bohlender et al. 1987). These techniques have been recently employed in studies by, e.g., Shorlin et al. (2002), Leone \& Catanzaro

$\dagger$ In fact, most modern methods employ a rotation of the retarder to permit switching of the positions of the two beams on the CCD, allowing the removal of systematic detector effects with multiple exposures; see, e.g., Semel et al. (1993).

$\ddagger$ Although limited in sensitivity to moderate $v \sin i$ objects, metallic line spectropolarimetry can exploit stellar rotation to allow the detection of magnetic fields even when the mean longitudinal magnetic field is null (see, e.g., Shorlin et al. 2002). 
(2004), Chadid et al. (2004), and Bagnulo et al. (2004), achieving in some cases precision better than 10 G. Notably, circular polarimetry of metallic lines by Babcock, Preston and collaborators, and in Balmer lines by Landstreet, Borra and collaborators, have led the way to the fundamental understanding of magnetism in intermediate mass stars that we enjoy today.

Fig. 5 illustrates the remarkable circular polarisation signatures detected in the lower Balmer series of the very young Rosette cluster member NGC 2244-334 (Bagnulo et al. 2004, observations using FORS1 at the ESO VLT).
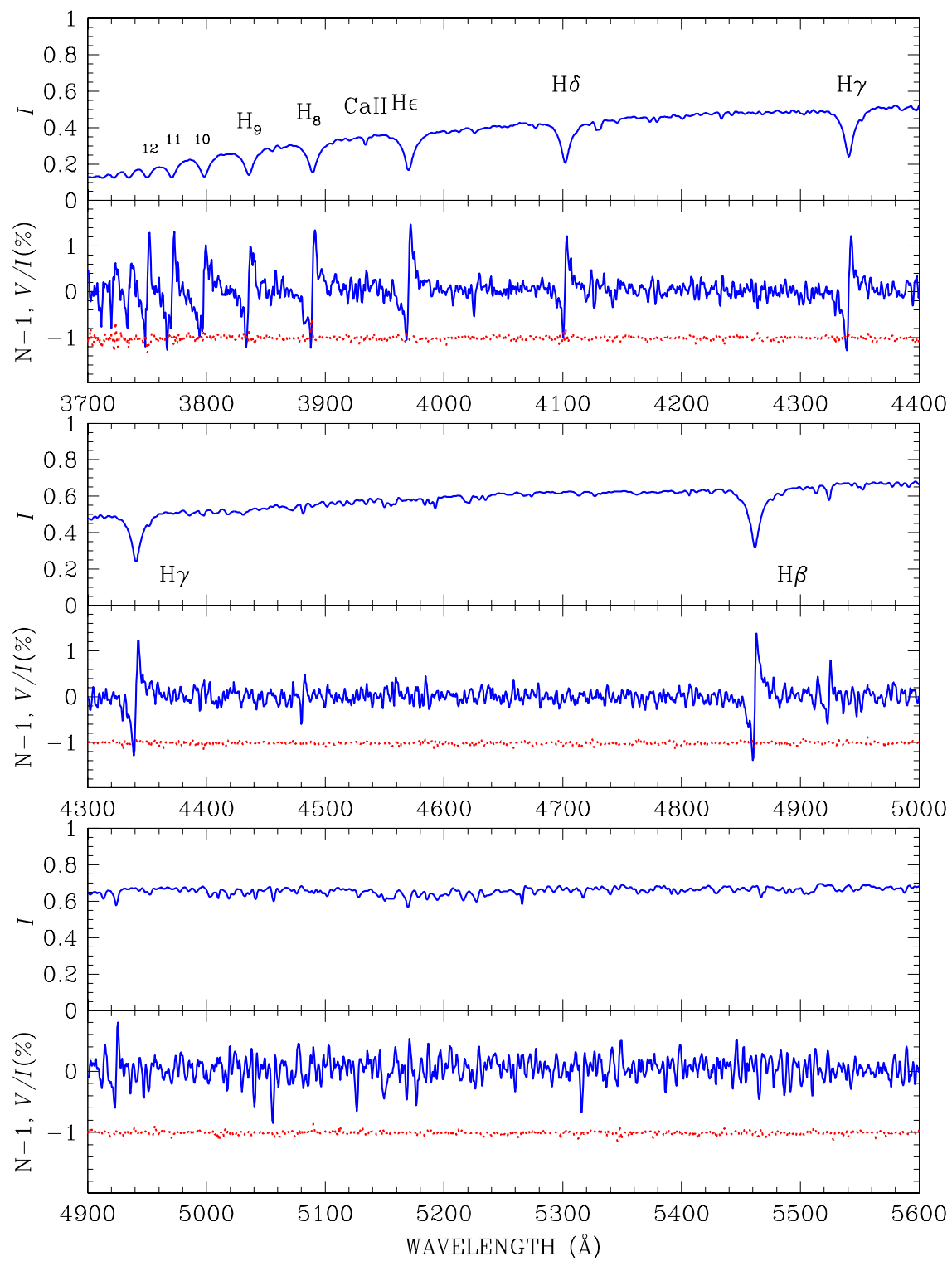

Figure 5. Stokes $I$ and $V$ spectra of the lower Balmer series of NGC 2244-334, obtained by Bagnulo et al. (2004) using the FORS1 spectropolarimeter at the ESO VLT. The longitudinal field diagnosed using these data is $-9 \mathrm{kG}$, the second-largest longitudinal field ever measured in a non-degenerate star. 


\subsection{Linear polarisation in the continuum and in metal lines}

By observing the transverse Zeeman effect (Zeeman linear polarisation) in combination with the circular polarisation longitudinal Zeeman effect, qualitatively new constraints on the magnetic field geometry of A stars can be obtained. The first systematic observations of the transverse Zeeman effect in A stars were obtained by Leroy and collaborators (e.g., Leroy 1995) during the early 1990s. These authors measured time variations of low levels of linear polarisation in the broadband light of Ap stars, produced as a result of the saturation of Zeeman-broadened metallic absorption lines. Upon comparison with the predictions of theoretical models, they found that the variations were not reproducible using symmetric magnetic field models. More recently, observations of transverse Zeeman effect within spectral absorption lines has become possible (Wade et al. 2000b obtained observations of several stars using the MuSiCoS spectropolarimeter at Pic du Midi observatory). Modeling of the phase variations of both circularly and linearly polarised line profiles for the Ap star 53 Cam has been performed using the Magnetic Doppler Imaging method (Kochukhov et al. y 2004) resulting in the first assumption-free high-resolution vector map of an Ap star magnetic field constructed from spectroscopic data in all four Stokes parameters. The maps of 53 Cam support the conclusions of Leroy and collaborators, revealing a remakably complex magnetic field: underlying dipolar characteristics, but with significant contributions by multipolar components of higher order.

\section{Indirect detection of magnetic fields}

Despite the flexibility and power of modern magnetic field diagnostic techniques, there remain stars in which magnetic fields cannot be detected directly, due to the limitations of the techniques themselves. Many of these limitations apply primarily to the hotter $\mathrm{B}$ and $\mathrm{O}$ type cousins of A stars; they are, however, important because of known or potential relationships between these objects and A stars.

Indirect methods for inferring fields in hard-to-study stars exploit phenomena typically associated with magnetic fields, such as surface structures, non-thermal radio, UV and X-ray emission, and azimuthal structuring of winds and other circumstellar matter. For example, the presence of periodic line profile variability in Main Sequence A stars, particularly when accompanied by particular patterns of photospheric abundance peculiarities (overabundances of rare eaths, Fe peak elements (especially $\mathrm{Cr}$ ) or $\mathrm{Si}$ ), are strongly indicative of the presence of magnetic fields (see, e.g,. Auriere \& Wade 2005). In A supergiants and hotter stars, variability of line profiles formed in stellar winds, along with non-thermal radio emission, UV line emission and X-ray emission, may indicate the influence of magnetic fields in imposing structure on stellar winds and other circumstellar matter and energy (e.g., Wade 2001). Finally, observations of Hanlé effect (e.g., Ignace 2003) may provide a new window to magnetic fields in wind-obscured stars. Many of these effects are only, or most effectively, observed from space.

\section{Conclusion}

Modern Zeeman magnetic field diagnostics provide a powerful and flexible suite of tools for studying magnetism in stellar atmospheres. The introduction of sensitive new spectropolarimeters on ground-based telescopes, both large (such as FORS1 at ESO/VLT, ESPaDOnS at CFHT) and small (SARG at TNG, NARVAL at TBL/Pic du Midi), as well as space-based platforms (the Far Ultraviolet Spectro-Polarimeter, FUSP) will provide a much more detailed picture of the characteristics of magnetic fields over a much larger fraction of all A stars. 


\section{References}

Auriere, M., Wade, G.A. 2005, These Proceedings, EP12

Bagnulo S., Szeifert T., Wade G.A., Landstreet J.D., Mathys G., 2002, A\&A 389, 191

Bagnulo S., Landstreet J.D., Lo Curto G., Szeifert T., Wade G.A., 2003, A\&A 403, 635

Bagnulo S., Hensberge H., Landstreet J.D., Szeifert T., Wade G.A., 2004, A\&A 416, 1149

Bohlender D.A., Landstreet J.D., Brown D.N., Thompson I.B., 1987, ApJ 323, 325

Chadid M., Wade G.A., Shorlin S.L.S., Landstreet J.D., 2003, A\&A 413, 1087

Donati J.-F., Semel M., Carter B.D., Rees D.E., Cameron A.C., 1997, MNRAS 291, 658

Ignace R., 2003, in the proceedings of Magnetic fields in $O, B$ and A stars, ASP conference series \#305, L. Balona, H.F. Henrichs \& R. Medupe (eds.), p.28

Kochukhov O., Bagnulo S., Wade G.A., Sangalli L., Piskunov N., Landstreet J. D., Petit P., Sigut T. A. A., 2004, A\&A 414,613

Landstreet J.D., 1988, ApJ 326, 967

Leone F. \& Catanzaro G., 2004, A\&A 425, 271

Leroy J.-L., 1995, A\&AS 114, 79

Mathys G. 1989, Fund. Cosmic Phys. 13, 143

Mathys G. 1995, A\&A 293, 746

Mathys G., Hubrig S., Landstreet J.D., Lanz T., Manfroid J., 1997, A\&AS 123, 353

Semel M., Donati J.-F., Rees D. E., 1993, A\&A 278, 231

Shorlin S.L.S., Wade G.A., Donati J.-F., Landstreet J.D., Petit P., Sigut T.A.A., Strasser S., 2002, A\&A 392, 637

Wade G.A., Donati J.-F., Landstreet J.D., Shorlin S.L.S., 2000a, MNRAS 313, 851

Wade G.A., Donati J.-F., Landstreet J.D., Shorlin S.L.S., 2000b, MNRAS 313, 823

Wade G.A., 2001, in the proceedings of Magnetic fields across the HR diagram, ASP conference series \# 248, G. Mathys \& S. Solanki (eds.), p. 403

Wade G.A., Bagnulo S., Kochukov O., Landstreet J.D., Piskunov N., Stift M.J., 2001, A\&A 374,265

Zeeman P., 1897, Nature 55, 347 